Proceedings of the 1996 IEEE

International Conference on Robotics and Automation

Minneapolis, Minnesota - April 1996

\title{
Location and Curvature Estimation of "Spherical" Targets using a Flexible Sonar Configuration *
}

\author{
Billur Barshan \\ Department of Electrical Engineering \\ Bilkent University \\ Bilkent, 06533 Ankara, Turkey
}

\begin{abstract}
A novel, flexible, three-dimensional (3-D) multisensor sonar system is employed to localize the center of a spherical target and estimate its radius of curvature. The interesting limiting cases for the problem under study are the point and planar targets, both of which are important for the characterization of a mobile robot's environment. A noise model is developed based on real sonar data. An extended Kalman filter (EKF) which incorporates the developed noise model is employed as an estimation tool for optimal processing of the sensor data. Simulations and experimental results are provided for specularly reflecting cylindrical targets.
\end{abstract}

\section{Introduction}

Although most of today's mobile robots are operating in $2 \frac{1}{2}-\mathrm{D}$ environments, $3-\mathrm{D}$ target recognition and discrimination of targets has potential significance for robots operating in 3-D environments such as airborne robots or robots designed for underwater applications. Recently, several researchers have started investigating the limitations of sonar for 3-D target recognition and tracking. In [1], the minimum amount of information and actuation needed to track a ball in 3-D has been determined and implemented using qualitative methods only, without quantifying and modeling any of the unknown parameters of the target. Hong and Kleeman have investigated the geometry of 3-D corner cubes using a low-sample rate equilateral triangular sonar configuration [2]. Kleeman and Akbarally have classified and discriminated the primitive target types commonly occurring in 3-D space [3]. Peremans et al [4] and Sabatini [5] have both investigated curved reflectors using a linear array configuration.

The aim of this paper is to estimate the unknown

${ }^{*}$ This work was supported by TÜBİTAK EEEAG-92 project and the British Council Academic Link Program. parameters of "spherical" targets in 3-D. In the next section, a detailed description of the sensing device is given. In Section 3, the geometry of reflection from a spherical target is considered and analyzed. The results for the limiting cases of point and planar targets are highlighted. In Section 4, these analytical results together with a correlated noise model are incorporated in an EKF to estimate target location and radius of curvature. Simulations and experimental results are provided in Section 5. Concluding remarks are made in the last section.

\section{The Sensing Device}

The sensing device used in this investigation was constructed very accurately for 3-D sonar applications. A trade-off between simplicity of mechanical design and flexibility was established. The unit consists of five Polaroid transducers, each operating at a resonant frequency of $f_{o}=49.4 \mathrm{kHz}$, where a single, central transducer is flanked by four receivers configured like the petals of a flower. The central transducer is fixed but the separation $d$ between the central transducer and each surrounding one can be manually adjusted to any value from 5 to $15 \mathrm{~cm}$. The device has 16 mechanical joints which enables it to move with the aid of a single stepper motor mounted behind the central transducer. In one extreme position, all the sensors are coplanar which corresponds to the reference point of the stepper motor. In the other extreme, the middle transducer is pulled back by $7.5 \mathrm{~cm}$ due to the displacement caused by turning of the stepper motor. These two extreme positions are illustrated in Figure 1a and b. In Figure 1b, the angle between the horizontal plane and the surrounding transducers is $30^{\circ}$. In between, the stepper motor can take 1020 steps with step size $0.9^{\circ}$. The flexibility of the sensor can be judicuously used in order to recognize 3-D targets and focus on them to extract valuable information from the sensed data. 


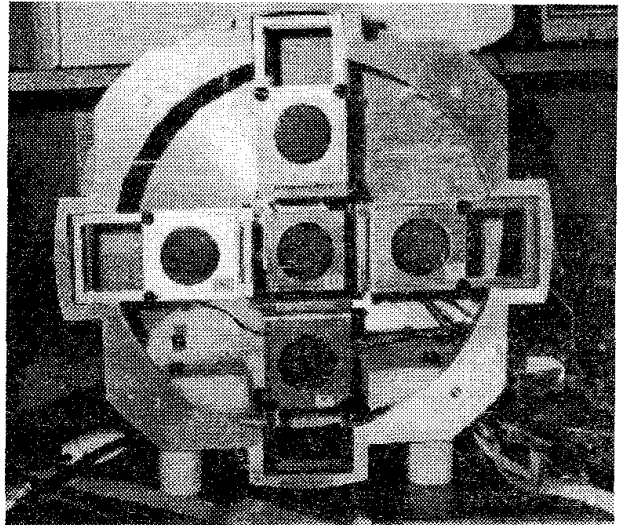

(a)

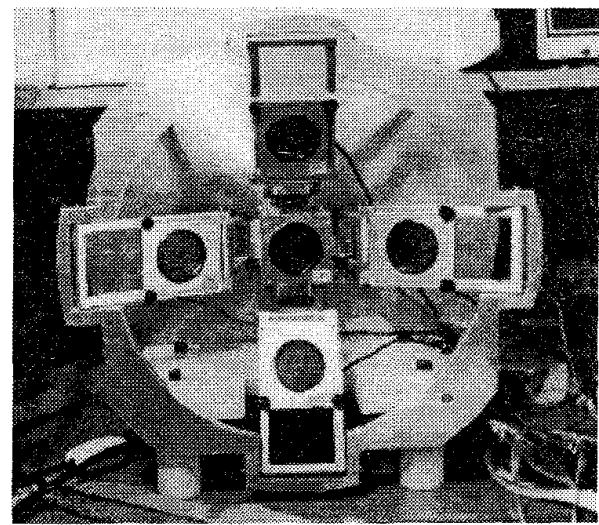

(b)

Figure 1: Two extreme positions of the sensing device.

The system is flexible enough that the transducers can be fired in any sequence. In the firing pattern selected, each transducer transmits and receives the echo of its own signal. Assuming the target is stationary, this can be done sequentially to avoid crosstalk between the transducers. After each transmission, the detected waveform is recorded and a TOF reading is obtained by thresholding.

\section{Target Reflection Geometry}

A stationary spherical target of radius $R$ is assumed to be present at spherical coordinates $(r, \theta, \phi)$.

\subsection{Spherical Target with radius $R$}

From the geometry of Figure 2, the round-trip distance measurements at the surrounding transducers are:

$$
h_{0}=\frac{c t_{o}}{2}=r-R
$$

$$
\begin{aligned}
& h_{r}=\frac{c t_{r}}{2}=\sqrt{r^{2}+d^{2}-2 d r \cos \phi \sin \theta}-R \\
& h_{l}=\frac{c t_{l}}{2}=\sqrt{r^{2}+d^{2}+2 d r \cos \phi \sin \theta}-R \\
& h_{u}=\frac{c t_{u}}{2}=\sqrt{r^{2}+d^{2}-2 d r \sin \phi}-R \\
& h_{d}=\frac{c t_{d}}{2}=\sqrt{r^{2}+d^{2}+2 d r \sin \phi}-R
\end{aligned}
$$

where $t_{o}, t_{r}, t_{l}, t_{u}, t_{d}$ are the TOF values from the transmitter to the middle, right, left, up and down transducers respectively, and $c$ is the speed of sound in free space. Each measurement confines the target location to a circle centered at the corresponding transducer. At least three measurements are ecessary to identify the curvature of the target both in 2-D and 3-D. This is illustrated in Figures 3 and 4. To localize the center of the target with finite radius, one needs three measurements in 2-D and five in $3-\mathrm{D}$ as indicated by the expressions for the true polar coordinates of the target:

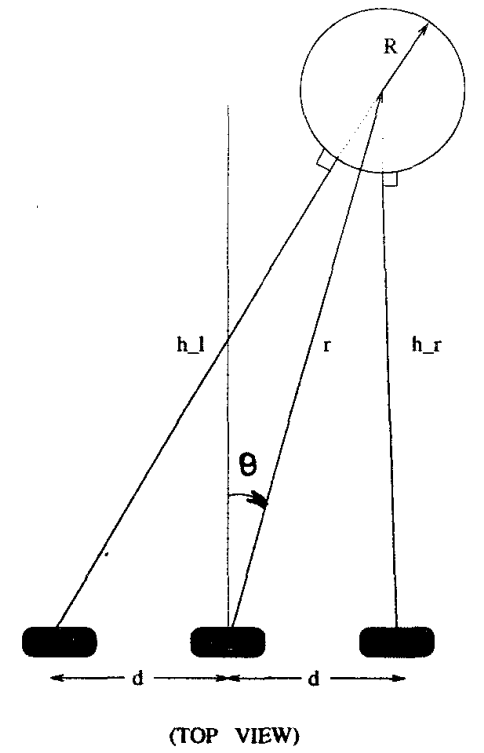

Figure 2: The geometry of the target position.

$$
\begin{aligned}
& R=\frac{\left(h_{r}^{2}+h_{l}^{2}\right)-2\left(h_{o}^{2}+d^{2}\right)}{4 h_{o}-2\left(h_{r}+h_{l}\right)}=\frac{\left(h_{u}^{2}+h_{d}^{2}\right)-2\left(h_{o}^{2}+d^{2}\right)}{4 h_{o}-2\left(h_{u}+h_{d}\right)} \\
& r=h_{o}+R \\
& \theta=\operatorname{asin} \frac{\left(h_{l}-h_{r}\right)\left(h_{l}+h_{r}+2 R\right)}{\sqrt{16 d^{2}\left(h_{o}+R\right)^{2}-\left(h_{d}-h_{u}\right)^{2}\left(h_{d}+h_{u}+2 R\right)^{2}}} \\
& \phi=\operatorname{asin} \frac{\left(h_{d}-h_{u}\right)\left(h_{d}+h_{u}+2 R\right)}{4 d\left(h_{o}+R\right)}
\end{aligned}
$$




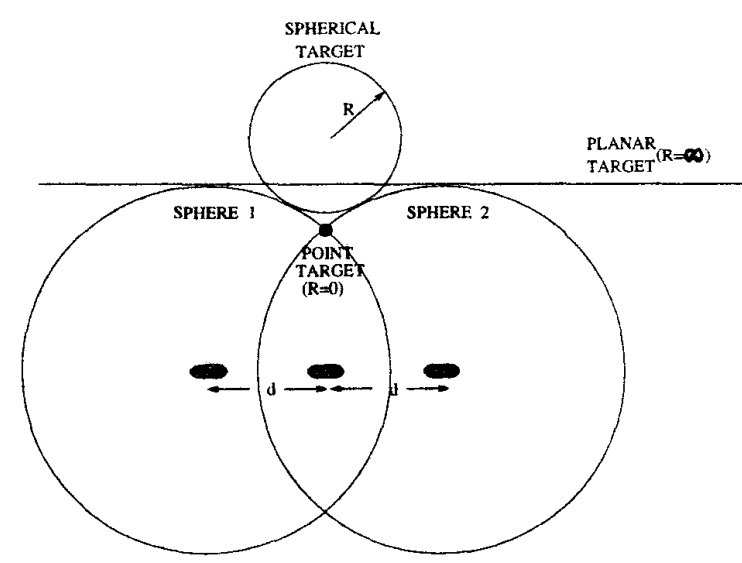

Figure 3: The indeterminacy of curvature with only two measurements. The unknown target can have any curvature from zero to infinity.

\subsection{Point Target: the limit $R \rightarrow 0$}

In the limit as $R \rightarrow 0$, a point target is obtained. Point target localization in 2-D has been considered in [6] and two methods of estimating the location have been presented using a linear array of transducers. The equations in 3-D derived above for finite $R$ become simpler in the limit as $R \rightarrow 0$ :

$$
\begin{aligned}
r & =h_{o}=\sqrt{\frac{h_{r}^{2}+h_{l}^{2}}{2}-d^{2}}=\sqrt{\frac{h_{u}^{2}+h_{d}^{2}}{2}-d^{2}} \\
\theta & =\operatorname{asin} \frac{\left(h_{l}^{2}-h_{r}^{2}\right)}{\sqrt{16 d^{2}\left(h_{o}+R\right)^{2}-\left(h_{d}^{2}-h_{u}^{2}\right)^{2}}} \\
\phi & =\operatorname{asin} \frac{\left(h_{d}^{2}-h_{u}^{2}\right)}{4 d\left(h_{o}+R\right)}
\end{aligned}
$$

Characterizing the point-target response of a sensor is important not only for its application to point targets but also to assess its performance on extended targets. There are different approaches to model extended targets in robotics applications $[2,4,7]$. If the approach is one of hypothesis testing or one of parametrizing the extended target, then sensor performance may not be easily related to its point-target response. On the other hand, for extended targets of unknown shape with possible roughness [8], point target analysis can be extremely useful.

\subsection{Planar Target: the limit $R \rightarrow \infty$}

For the limiting case of $R \rightarrow \infty$, the target becomes a plane. Both the distance to the center of the "sphere" and its radius of curvature become infinity. In this case, either the limits of the above equations can be taken carefully or, more simply, the perpendicular distances of the transducers to the plane can be
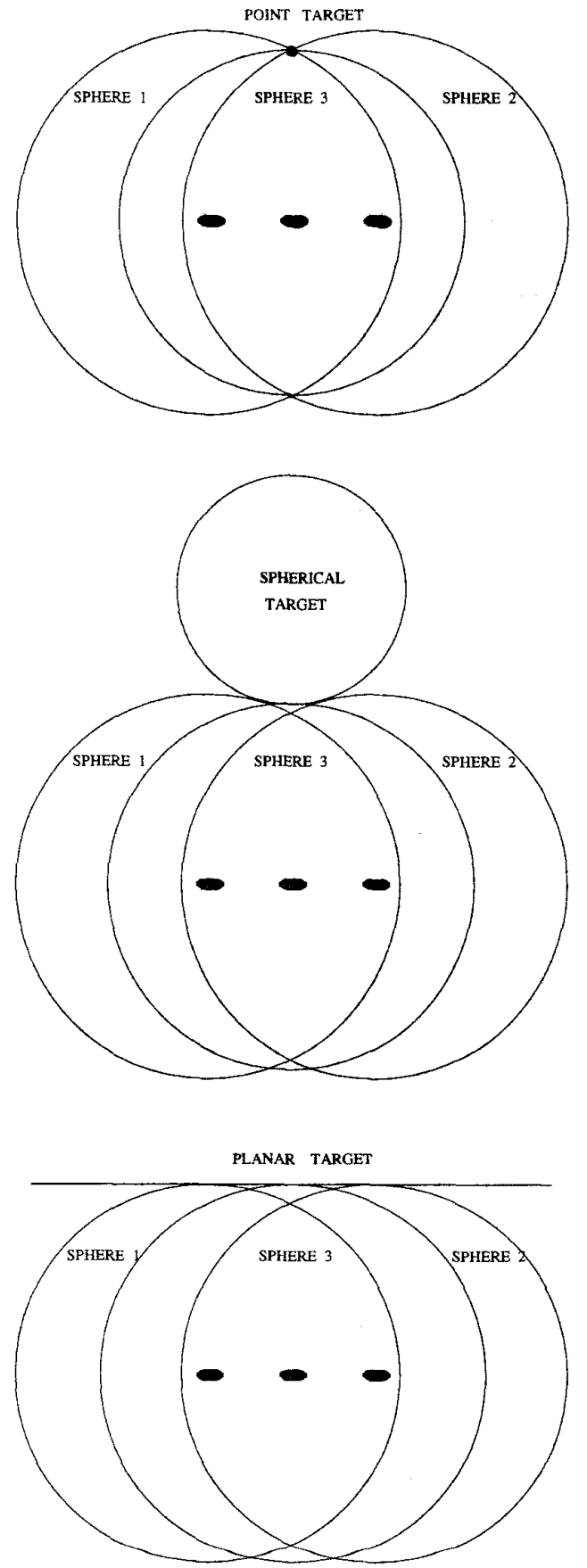

Figure 4: The geometry of point, spherical and planar targets for three measurements. Three measurements uniquely identify the curvature of the unknown target both in $2-\mathrm{D}$ and $3-\mathrm{D}$. 


\begin{tabular}{||r||r||r||r||}
\hline \hline & \multicolumn{1}{|c|}{$R=5 \mathrm{~cm}$} & $R=30 \mathrm{~cm}$ & $R=\infty$ \\
\hline \hline$E[R]$ & $4.82 \mathrm{~cm}$ & $30.4 \mathrm{~cm}$ & $2790 \mathrm{~cm}$ \\
\hline$E_{\mathrm{EKF}}[R]$ & $4.98 \mathrm{~cm}$ & $30.1 \mathrm{~cm}$ & $3946 \mathrm{~cm}$ \\
\hline$\sigma_{R}$ & $1.17 \mathrm{~cm}$ & $1.23 \mathrm{~cm}$ & $1.19 \mathrm{~cm}$ \\
\hline$E[r]$ & $99.6 \mathrm{~cm}$ & $100.2 \mathrm{~cm}$ & $100.3 \mathrm{~cm}$ \\
\hline$E_{\mathrm{EKF}}[r]$ & $99.9 \mathrm{~cm}$ & $100.1 \mathrm{~cm}$ & $100.1 \mathrm{~cm}$ \\
\hline$\sigma_{r}$ & $1.28 \mathrm{~cm}$ & $1.48 \mathrm{~cm}$ & $1.35 \mathrm{~cm}$ \\
\hline$E[\theta]$ & $0.32^{\circ}$ & $0.03^{\circ}$ & $-0.48^{\circ}$ \\
\hline$E_{\mathrm{EKF}}[\theta]$ & $0.09^{\circ}$ & $0.04^{\circ}$ & $-0.22^{\circ}$ \\
\hline$\sigma_{\theta}$ & $0.24^{\circ}$ & $0.33^{\circ}$ & $0.54^{\circ}$ \\
\hline
\end{tabular}

Table 1: Experimental results.

directly derived from the geometry as:

$$
\begin{aligned}
h_{r} & =h_{o}-d \cos \phi \sin \theta \\
h_{l} & =h_{o}+d \cos \phi \sin \theta \\
h_{u} & =h_{o}-d \sin \phi \\
h_{d} & =h_{o}+d \sin \phi
\end{aligned}
$$

The solution is:

$$
\begin{aligned}
r & =h_{0}+R=\infty \\
\theta & =\operatorname{asin} \frac{h_{l}-h_{r}}{\sqrt{4 d^{2}-\left(h_{d}-h_{u}\right)^{2}}} \\
\phi & =\operatorname{asin} \frac{h_{d}-h_{u}}{2 d}
\end{aligned}
$$

The distance to the surface of the plane is simply $h_{o}$.

\section{Estimation of Target Position}

In real sonar systems, there are three principal components of noise, which are thermal noise in the receivers, variations in the velocity of sound $c$, and acoustic amplitude fluctuations due to air-temperature variations [5]. These effects can be effectively modeled by two different noise components: First, there is the rapidly varying and uncorrelated noise component, which is amplitude dependent. It represents the errors due to quantization of the received waveforms, timesampling of the signal and thermal noise of receiving circuit electronics. The second noise component varies more slowly in time compared to the first, and is highly correlated. The main contributing factor is the momentary fluctuations of the speed of sound in air due to temperature, humidity changes and drafts of air, encountered very frequently in typical indoor environments. Such macroscopic environmental changes affect all five measured distances similarly, causing spatial correlation between the receivers.

These effects were modeled by using a covariance matrix with constant spatial correlation between the measurements. With the additive noise incorporated, the noiseless measurement equations given in Equations 1 become:

$$
\mathbf{z}(k)=\mathbf{h}[\boldsymbol{\Phi}(k)]+\mathbf{v}(k)
$$

where $\mathbf{v}(k)$ is the measurement noise process vector. Since the observation model is nonlinear, an EKF is used to filter the noisy data and obtain estimates for $\boldsymbol{\Phi}=(R, r, \theta, \phi)^{T}$. A first-order approximation to the function $\mathbf{h}($.) is made by first finding its Jacobian $\nabla \mathbf{h}$. This is used to update the covariance matrices as described in [9].

The states that need to be estimated are the true values of $R, r, \theta$ and $\phi$. The resulting state equations for a stationary target are as follows:

$$
\left[\begin{array}{c}
R(k+1) \\
r(k+1) \\
\theta(k+1) \\
\phi(k+1)
\end{array}\right]=\left[\begin{array}{c}
R(k) \\
r(k) \\
\theta(k) \\
\phi(k)
\end{array}\right]+\left[\begin{array}{c}
w_{R}(k) \\
w_{r}(k) \\
w_{\theta}(k) \\
w_{\phi}(k)
\end{array}\right]
$$

or in matrix notation:

$$
\mathbf{\Phi}(k+1)=\mathbf{\Phi}(k)+\mathbf{w}(k) .
$$

The process noise represents any deviations from the assumed model of state evolution, described by the state equation above, and is assumed to be zeromean and white. The state vector estimated by the filter is given by

$$
\hat{\mathbf{\Phi}}(k+1 \mid k+1)=\hat{\mathbf{\Phi}}(k \mid k)+\mathbf{W}(k+1) \nu(k+1)
$$

where $\mathbf{W}(k+1)$ is the filter gain, and $\nu(k+1)=$ $\mathbf{z}(k+1)-\mathbf{h}[\hat{\mathbf{\Phi}}(\mathbf{k}+\mathbf{1} \mid \mathbf{k})]$ is the innovations vector provided by the new observation at time $(k+1) T_{s}$.

\section{Results}

The sensor is mounted on the rotating turret of a Nomad 200 mobile robot. The rotational capability of the turret is to be used in the next stage of this study to track targets on the horizontal plane. Data from the sonar sensors was collected using a PCL-816 $\mathrm{A} / \mathrm{D}$ card with 12 bit resolution and was processed by an IBM-PC 486 using the $\mathrm{C}$ language. The EKF was implemented in real time on the same system. A target was placed at $\left(r=100 \mathrm{~cm}, \theta=0^{\circ}\right)$ in front of the sensing system with $d=15 \mathrm{~cm}$. The measurement noise standard deviation was experimentally determined to be $0.17 \mathrm{~cm}$. For each target, a set of 1000 measurements were collected and filtered by the EKF to obtain the minimum mean square estimates of $R, r, \theta, \phi$. Meanwhile, the raw data was used at each step to obtain estimates of the state variables without 
filtering. The expected value and standard deviations of these estimates were computed over the same 1000 realizations. In Table 1, experimental results for three different targets are displayed, which represent point, cylidrical and planar targets. In Figures 5 and 6 , results of a Monte Carlo study with 1000 realizations over a wider parameter range are illustrated. In obtaining these results, the attenuation of the signal amplitude was not considered to isolate the effect of the system configuration. In these results, whenever other parameters are varied, $\theta$ and $\phi$ were kept constant at $0^{\circ}$. When $\theta$ and $\phi$ were varied, their maximum values were limited to $\pm 15^{\circ}$ since this is the approximate beamwidth of the mainlobe of the Polaroid transducer. Similarly, $r$ was set to $100 \mathrm{~cm}, R=5 \mathrm{~cm}$ and $d=15$ $\mathrm{cm}$ unless where they were varied. In Figure 5 (a), the effect of increasing the standard deviation of the measurement noise standard deviation is illustrated. The solid lines indicate the mean values and the dashed lines correspond to the one standard deviation interval around the mean. As expected, increasing the measurement noise increases the standard deviations of the estimated parameters. In Figure 5 (b) and (c) the effect of varying $R$ and $r$ is seen on the accuracy of the other parameters. Varying the radius of the target does not seem to affect positional accuracy. The results for $E[\phi]$ are almost identical to $E[\theta]$, hence, they were not included here for brevity.

In Figure 6 (a), the results for varying $\theta$ are given. Increasing $\theta$ increases the estimation error on $\theta$ but does not affect the accuracy of the remaining parameters since the attenuation in the signal amplitude is not considered. Same is true for $\phi$, and again, the results are not included here for brevity. In Figure 6 (b), the effect of varying $d$ is illustrated. Increasing the transducer separation provides better resolution of the target parameters and reduces the standard deviation of the estimated parameters. If the target radius is much smaller compared to transducer separation, some of the sensors will not be able to receive an echo from the target, setting an upper bound on $d$.

\section{Conclusion}

A sensing device capable of estimating the location and radius of curvature of spherical and cylindrical targets has been described. Analytical results are verified by simulations and real data from cylindrical targets. Typical accuracies are less than $0.5 \mathrm{~cm}$ in curvature and range, and less than $1^{\circ}$ in angle. The two limits of interest are the point (3-D) or line (2-D) target and the planar target. The next stage of this work is to track cylindrical targets in azimuth. A future more in- telligent sensor could also have the capability to track in elevation to add more flexibility to the application.

\section{References}

[1] R. Kuc "Three-dimensional tracking using qualitative bionic sonar," Robotics and Autonomous Systems, vol. 11, pp. 213-219, 1993.

[2] M. L. Hong and L. Kleeman "Analysis of ultrasonic differentiation of three-dimensional corners, edges and planes," in Proceedings IEEE International Conference on Robotics and Automation, pp. 580 584, Nice, France, May 12-14, 1992.

[3] L. Kleeman and H. Akbarally "A sonar sensor for accurate 3d target localization and classification," in Proceedings IEEE International Conference on Robotics and Automation, pp. xxx-xxx, Nagoya, Japan, May 21-27, 1995.

[4] H. Peremans, K. Audenaert and J. M. Van Campenhout "A high-resolution sensor based on triaural perception," IEEE Transactions on Robotics and Automation, vol. 9, pp. 36-48, February 1993.

[5] A. M. Sabatini "Statistical estimation algorithms for ultrasonic detection of surface features," in Proceedings IEEE/RSJ International Conference on Intelligent Robots and Systems, pp. 1845-1852, Munich, Germany, September 12-16, 1994.

[6] B. Barshan and O. Arıkan "Performance analysis of two linear array processing algorithms for pointobstacle localization," in Proceedings SPIE Signal and Data Processing of Small Targets, pp. $\mathrm{xxx}-$ xxx, San Diego, CA, July 11-13, 1995.

[7] J. J. Leonard and H. F. Durrant-Whyte "Mobile robot localization by tracking geometric beacons," IEEE Transactions on Robotics and Automation, vol. 7, pp. 376-382, 1991.

[8] Ö. Bozma and R. Kuc "Characterizing pulses reflected from rough surfaces using ultrasound," Journal of the Acoustical Society of America, vol. 89, pp. 2519-2531, June 1991.

[9] Y. Bar-Shalom and T. E. Fortmann. Tracking and Data Association. Academic Press, New York, NY, 1988. 

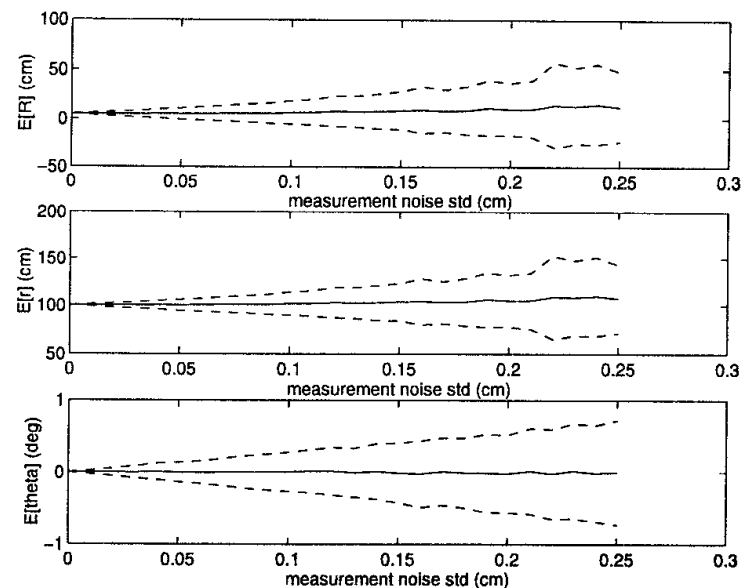

(a)
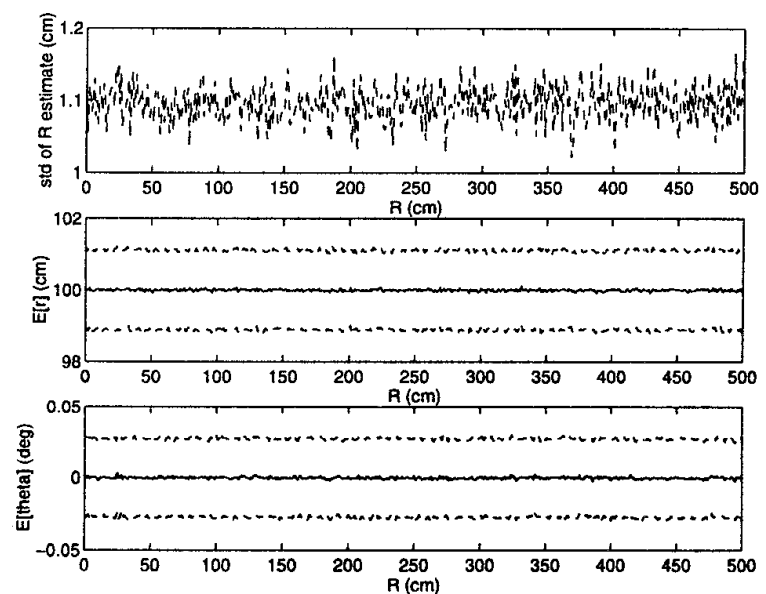

(b)
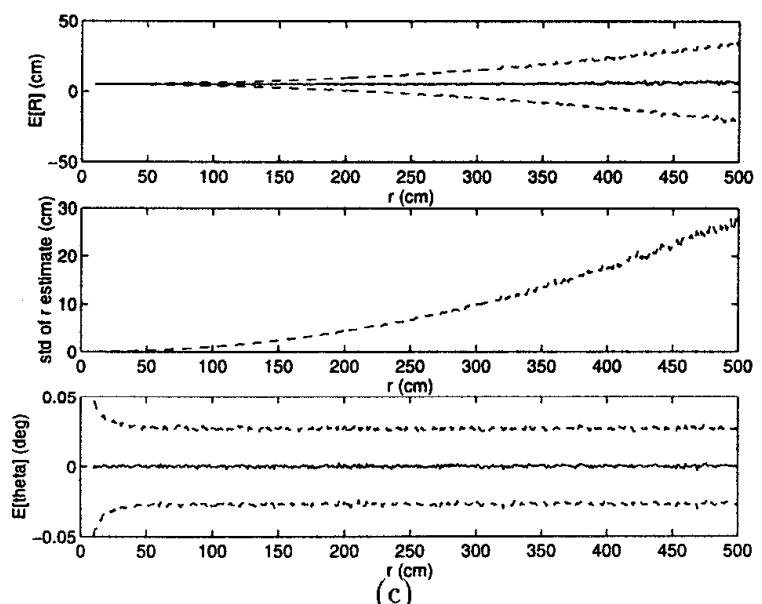

Figure 5: Results showing the variation of mean and standard deviation of the estimated parameters with measurement noise, $R$ and $r$.
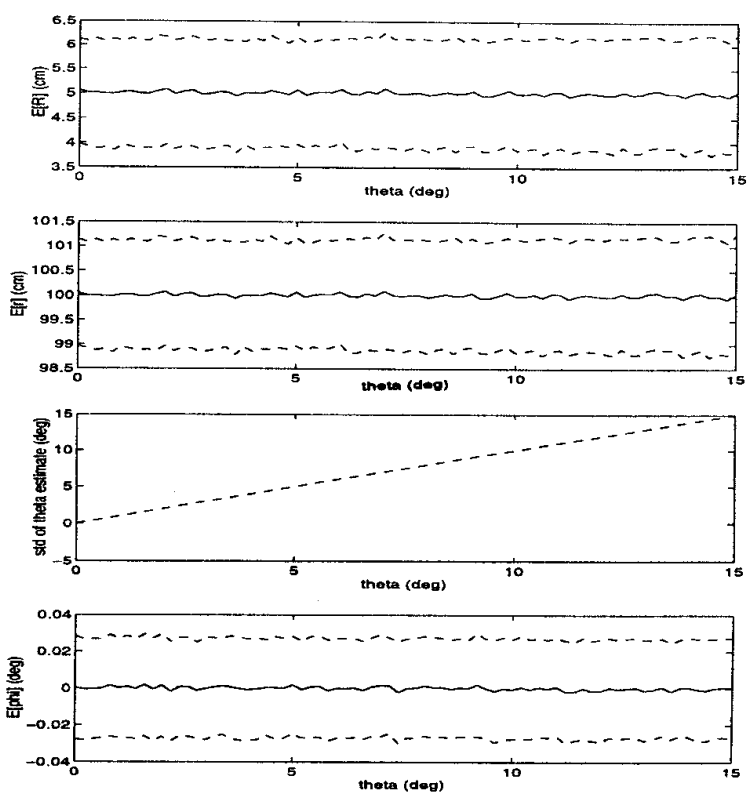

(a)
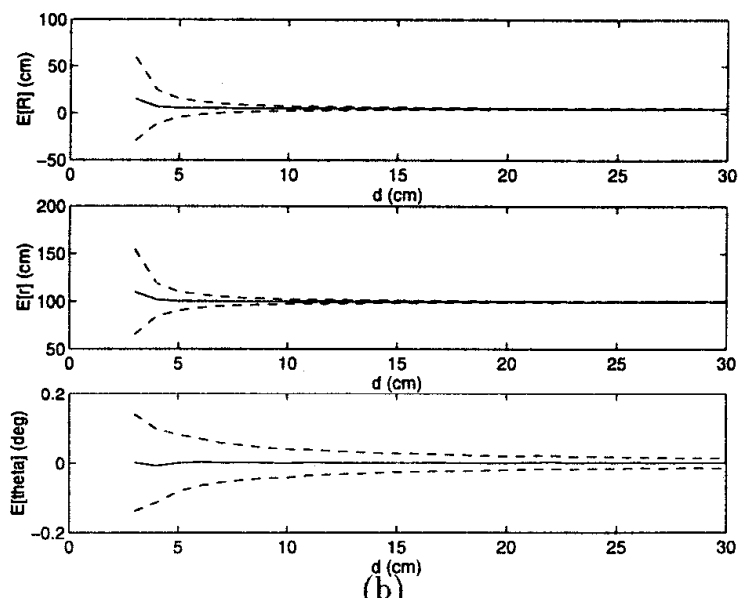

Figure 6: Results showing the variation of mean and standard deviation of estimated parameters with $\theta$ and transducer separation $d$. 